\title{
Heat Load Influence on Supermodes in Yb-Doped Four-Core Fibers
}

\author{
Poli, Federica; Lægsgaard, Jesper; Cucinotta, Annamaria; Selleri, Stefano
}

Published in:

Journal of Lightwave Technology

Link to article, DOI:

$10.1109 / J L T .2020 .3026618$

Publication date:

2021

Document Version

Peer reviewed version

Link back to DTU Orbit

Citation (APA):

Poli, F., Lægsgaard, J., Cucinotta, A., \& Selleri, S. (2021). Heat Load Influence on Supermodes in Yb-Doped Four-Core Fibers. Journal of Lightwave Technology, 39(1), 263-269. https://doi.org/10.1109/JLT.2020.3026618

\section{General rights}

Copyright and moral rights for the publications made accessible in the public portal are retained by the authors and/or other copyright owners and it is a condition of accessing publications that users recognise and abide by the legal requirements associated with these rights.

- Users may download and print one copy of any publication from the public portal for the purpose of private study or research.

- You may not further distribute the material or use it for any profit-making activity or commercial gain

- You may freely distribute the URL identifying the publication in the public portal

If you believe that this document breaches copyright please contact us providing details, and we will remove access to the work immediately and investigate your claim. 


\title{
Heat Load Influence on Supermodes in Yb-Doped Four-Core Fibers
}

\author{
Federica Poli, Member, IEEE, Jesper Lægsgaard, Annamaria Cucinotta, Member, IEEE, \\ and Stefano Selleri, Senior Member, IEEE
}

\begin{abstract}
Multicore fibers have been recently proposed as a possible solution to overcome the problems which affect fiberbased lasers and amplifiers when their output power increases, especially the severe power limitations imposed by thermal effects, responsible of the detrimental phenomenon of mode instability. In this paper the influence of the heat load generated in the Ybdoped cores of four-core fibers on the guiding properties of the supermodes is analyzed. Effective area and single-mode regime are calculated for fibers with different core-to-core separations, in order to identify the most convenient optical coupling regime among the cores in the presence of strong thermal effects. Moreover, through the comparison with dual-core fibers when the same total heat load is generated, useful guidelines for the choice of core number in multicore fiber cross-section are provided. Results show that there is a trade-off between effective area and single-mode regime, because four-core fibers provide the largest effective area, but they become multimode when thermal effects are significant, especially if the cores are optically coupled. When the distance among the cores is large enough, multicore fibers demonstrate to be less affected by the generated heat load, regardless of the number of the doped cores in the transverse section.
\end{abstract}

\section{INTRODUCTION}

$\mathbf{M}$ ULTICORE fibers represent a hot research topic nowadays, due to the potential advantages offered for many applications, ranging from terrestrial [1] and submarine [2] communications based on spatial division multiplexing techniques, to different sensing technologies [3] and optical amplification [4]. In particular, fibers with more than one Yb-doped core placed in the cross-section [5], [6] provide a possible solution for further power scaling of short and ultra-short pulsed fiber lasers, thus overcoming the limits due to nonlinear and thermal effects [7]. In fact, it has been demonstrated that it is possible to go beyond the power limit of a single amplifier through the coherent combination of multiple fiber amplifiers [8], [9]. With multicore fibers the main problem of this approach, that is the linear increase of the required components with the number of combined beams, can be mitigated [10]-[12]. Moreover, the amplification in fibers with doped cores close enough to be coupled can exploit an intrinsic coherence property, which can be automatically maintained along the amplifier without any feedback system, since the light propagates as supermodes (SMs) [4].

Multicore fibers can have a potentially positive impact also

F. Poli, A. Cucinotta and S. Selleri are with the Department of Engineering and Architecture, University of Parma, I-43124 Parma, Italy, e-mail: federica.poli@unipr.it.

J. Lægsgaard is with DTU Fotonik, Department of Photonics Engineering, Technical University of Denmark, DK-2800 Kongens Lyngby, Denmark. on transverse mode-instability (TMI), a thermally-induced phenomenon which is detrimental for the performances of high-power fiber lasers, since it destroys the quality and the stability of the laser beam when a certain average power threshold is reached [13]. Experiments made on a multicore fiber with a row of four Yb-doped cores have demonstrated that the TMI threshold is four times higher than the one of the single-core fiber, thus showing a linear increase with the core number [5]. This result has been obtained for a fiber with optically decoupled cores, that is with a large separation, and designed to provide a tight field confinement [5].

It is not straightforward to extend the same conclusion about TMI threshold to multicore fibers with a significant optical coupling among the cores, since in this situation it is necessary to take into account also the thermal coupling among supermodes. In recent theoretical works thermo-optic instabilities have been studied in dual-core fiber amplifiers [14], [15], showing a significantly different behaviour according to the supermode selected for the amplification. In particular, a TMI threshold increase with the core separation has been demonstrated, if the odd supermode is chosen for amplification [15]. Analogously, the selective excitation and the stable amplification of the out-of-phase supermode, which offers stability at arbitrary high power levels, unlike the fundamental one, have been experimentally and numerically demonstrated in an $\mathrm{Yb}$-doped multicore fiber with six coupled cores arranged in a ring [4].

In this work the influence of thermal effects on the supermode propagation in multicore fibers with four $\mathrm{Yb}$-doped cores, arranged in a square with variable side dimension, that is with different optical coupling conditions, is numerically analyzed through the finite element method (FEM). Being the scaling of the total active core area essential for increasing the pulse energy and the peak power in fiber-based amplifiers [9], the study recently done for $\mathrm{Yb}$-doped dual-core fibers [16] is extended to the case of four active cores in the fiber cross-section. Since the thermally-induced refractive index change in the doped cores provides a tighter field confinement of the supermodes, the beaviour of effective area and single-mode regime is analyzed as a function of the total heat load generated as sideeffect of the amplification process [17]. In fact, as it happens in single-core fibers, thermal effects can be responsible for the propagation also of higher-order modes (HOMs) in the originally single-mode cores of multicore fibers, thus causing a decrease of the potential amplifier combination efficiency [18]. Simulation results have demonstrated that four-core fibers can provide a larger effective area if the fundamental supermode is 


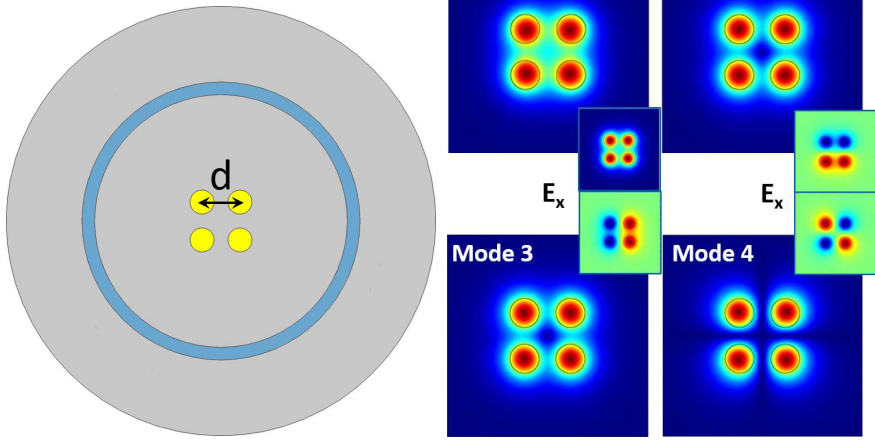

Fig. 1. (Left) Cross-section of 4-core fibers with different center-to-center distance $d$ between two doped cores (yellow regions). The light-blue ring is the air layer surrounding the inner cladding. (Right) Electric field modulus distribution of 4 fundamental SMs for the fiber with $V$-parameter $=1.735$ and $d=30 \mu \mathrm{m}$. The corresponding electric field $x$ component distribution is shown in smaller figures.

considered, especially when the cores are close enough to be optically coupled. However, for short core-to-core separations, if the core number in the fiber cross-section increases from two to four, under the same total heat load, the single-mode regime is more affected by thermal effects. On the contrary, the robustness of the single-mode behaviour to the generated heat load is higher for both two- and four-core fibers if the active cores are far from each other, that is optically decoupled.

\section{YB-DOPED 4-CORE FIBERS}

The characteristics of the $\mathrm{Yb}$-doped 4-core fibers studied in this analysis are analogous to the ones of the dual-core fibers considered in [16]. In particular, for each core the diameter is fixed to $19 \mu \mathrm{m}$ and the refractive index is properly modified to obtain the $V$-parameter equal to $1.735,2.2$ and 2.4 , while keeping fixed the silica refractive index for the cladding, thus providing an intrinsically single-mode behaviour at the signal wavelength of $1032 \mathrm{~nm}$. In this way, it is possible to compare immediately the behaviour of the two types of multicore fibers, as well as to deduce useful guidelines on the impact of core number on the performances of amplifiers based on this kind of fibers. As shown in Fig. 1(left), the four Yb-doped cores are placed at the vertices of a square of side $d$, varied in the range between $20 \mu \mathrm{m}$ and $65 \mu \mathrm{m}$. The outer part of the 4-core fiber cross-section is the same described in [16]. In particular, we consider an outer diameter sufficiently large to render the fiber unbendable, a so-called rod-type fiber. This serves to minimize random couplings between supermodes, which is undesirable for high-power single-mode amplification, although it has been actively exploited for amplifiers working at telecom wavelengths [19].

A FEM-based full-vector modal solver [20] has been used to calculate the modes of the multicore fibers when different optical coupling regimes are considered, by changing the coreto-core distance and the $V$-parameter. Four fundamental SMs have been evaluated in the present analysis [21], [22], labelled from Mode 1 to Mode 4 as the effective index $n_{\text {eff }}$ decreases. Notice that Mode 2 and Mode 3, with an intermediate $n_{e f f}$ value, are degenerate. Fig. 1(right) reports the distributions of

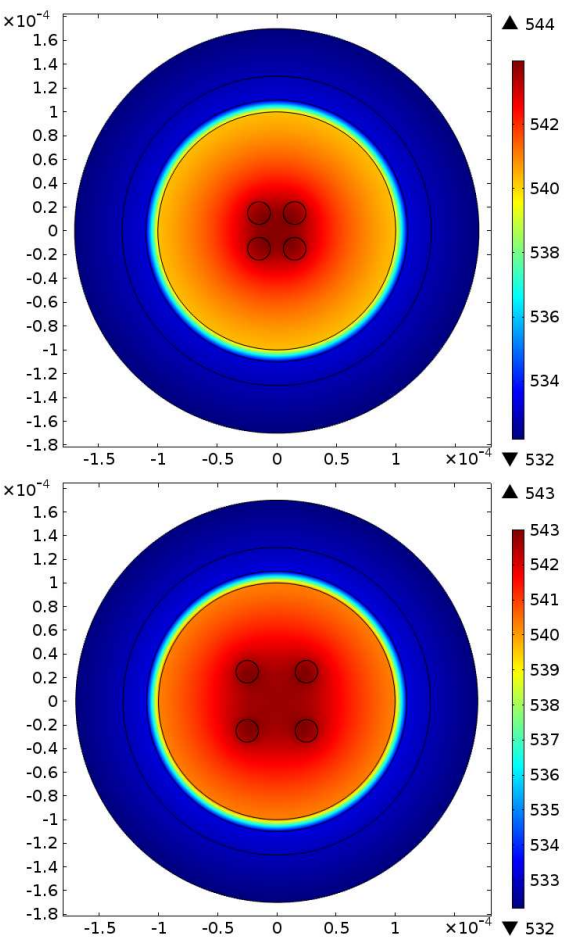

Fig. 2. Temperature distribution on the cross-section of the 4-core fibers with $V$-parameter $=1.735$ and, respectively, (top) $d=30 \mu \mathrm{m}$, and (bottom) $d=$ $50 \mu \mathrm{m}$, when $q_{\text {tot }}=20 \mathrm{~W} / \mathrm{m}$.

the modulus and the $x$ component of the electric field for the fundamental SMs in the fiber with $V$-parameter $=1.735$ and $d$ $=30 \mu \mathrm{m}$. The transverse components of the electric field are useful to identify the different fundamental supermodes. The $y$ component is not reported in the figure, being dual to the $x$ one.

The heat generation in the Yb-doped cores has been taken into account through the thermal model described in [16]. In particular, a total heat load $q_{t o t}$, uniformly distributed among the cores, so that $q_{t o t}=q \times$ core number, has been considered for the simulations. Notice that an uniform distribution of signal intensity over the cores has been taken into account, such as when a single supermode is excited, and this justifies the symmetric heat load we consider, which maintains the symmetry of the modal solutions. As shown by couple-mode results for dual-core amplifiers in [14], [15], this symmetry may not be stable, but this kind of modeling is outside the scope of the present paper. The heat generated in the cores due to the quantum defect causes a temperature increase, as demonstrated by Fig. 2(top) and (bottom), which show, respectively, the temperature distribution in the transverse section of the fibers with $V$-parameter $=1.735$, and with $d$ $=30 \mu \mathrm{m}$ and $d=50 \mu \mathrm{m}$ when $q_{t o t}=20 \mathrm{~W} / \mathrm{m}$. Notice that the raise is not only in the core areas, but in all the fiber cross-section, and especially in the central region bounded by the four cores. As a consequence, the thermo-optic effect significantly modifies the fiber refractive index profile in all the transverse cross-section [16], [23]. 

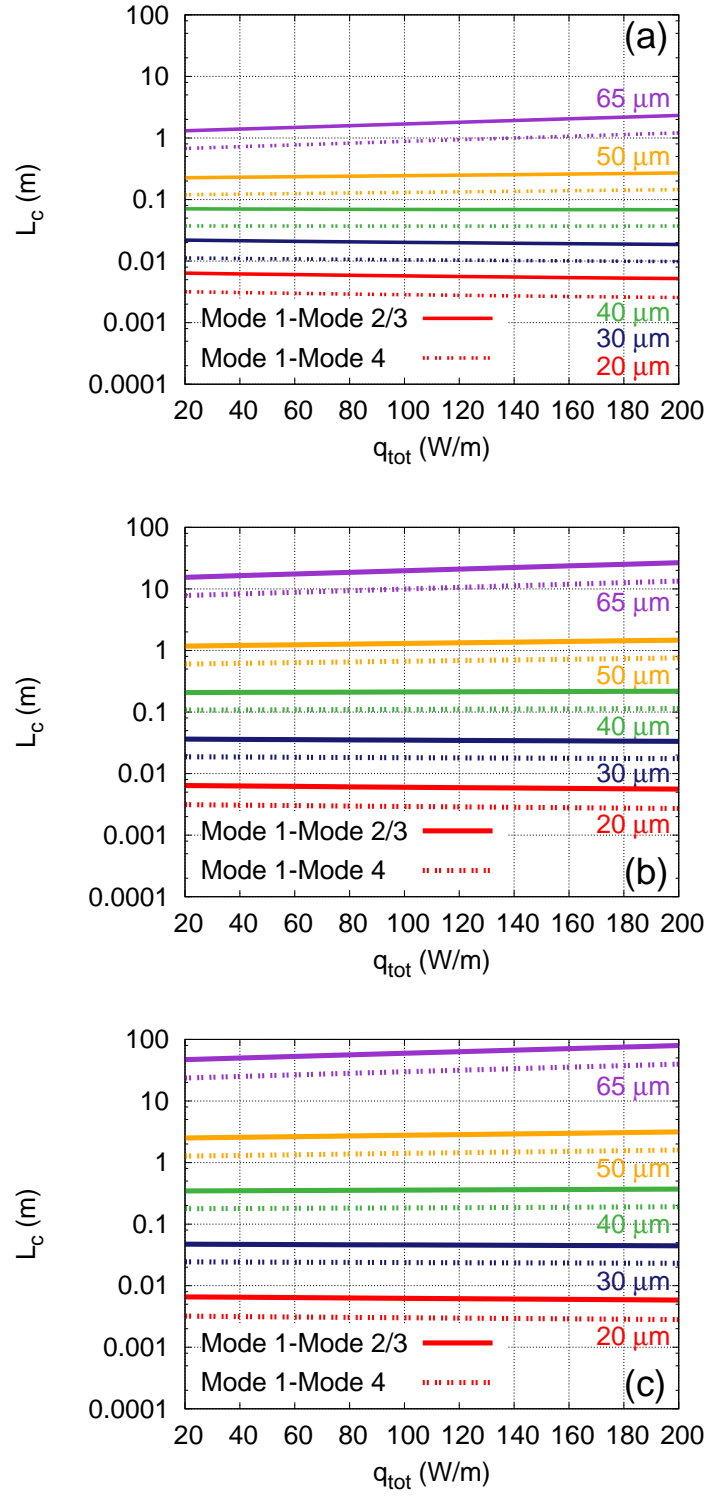

Fig. 3. Coupling length $L_{c}$ between fundamental SMs as a function of $q_{t o t}$ for different distance $d$ for 4-core fibers with $V$-parameter equal to (a) 1.735 , (b) 2.2 and (c) 2.4 .

\section{HeAt LoAd InfLUENCE}

In order to demonstrate that the influence of the thermal effects on the supermodes is different according to the core optical coupling conditions, the coupling length $L_{c}$ [16] between two fundamental modes of the 4-core fibers has been calculated. Fig. 3(a), (b) and (c) report the $L_{c}$ values obtained for the fibers with $V$-parameter equal to $1.735,2.2$ and 2.4, respectively, for the minimum and maximum core separation considered in this analysis, that is $20 \mu \mathrm{m}$ and $65 \mu \mathrm{m}$, and for some intermediate values, that is $d=30,40,50 \mu \mathrm{m}$, as a function of $q_{t o t}$ in the range between $20 \mathrm{~W} / \mathrm{m}$ and 200 $\mathrm{W} / \mathrm{m}$. The coupling length has been calculated for two couples of fundamental SMs, taking Mode 1 as a reference. Due to their degeneracy, which is preserved also under heat load in fibers wihtout asymmetries in geometric and dieletric core properties, Mode 2 and Mode 3 are considered as a unique supermode, labelled as Mode 2/3 in the figure legend. Notice that for the fiber with the lowest $V$-parameter, as $q_{t o t}$ becomes higher, $L_{c}$ decreases for both the SM couples when a coreto-core distance $d \leq 40 \mu \mathrm{m}$ is considered. On the contrary, it increases for higher $d$ values, that is for $50 \mu \mathrm{m}$ and 65 $\mu \mathrm{m}$, thus showing a weaker coupling among the cores. Since the slope of the coupling length versus the total heat load is negative, but very small for the SMs of the fiber with $d=40$ $\mu \mathrm{m}$, it is possible to conclude that $L_{c}$ is almost unaffected by $q_{\text {tot }}$ if the core separation is slightly larger than $40 \mu \mathrm{m}$. Notice that this $L_{c}$ behaviour is analogous to the one obtained for the dual-core fibers, taking into account even and odd fundamental SMs [16]. It is interesting to underline that the increase of the core number from two to four in multicore fibers with $V$-parameter equal to 1.73 under the same $q_{t o t}$, enlarges of about $10 \mu \mathrm{m}$, from $\sim 30 \mu \mathrm{m}$ [16] to $\sim 40 \mu \mathrm{m}$, the core-to-core distance corresponding to a negligible influence of thermal effects on the core coupling. Finally, it is important to notice that only the SMs of the 4-core fiber with $d=65 \mu \mathrm{m}$ can be considered optically decoupled, being their $L_{c}$ higher than the typical length of rod-type fiber amplifiers, that is $0.8 \mathrm{~m}$. In particular, for this core separation the coupling length of Mode 1 and Mode 2/3 is larger than $0.8 \mathrm{~m}$ whatever the $q_{\text {tot }}$ value in the range $20 \div 200 \mathrm{~W} / \mathrm{m}$, while Mode 1 and Mode 4 are optically decoupled only if the total heat load is higher than $60 \mathrm{~W} / \mathrm{m}$.

When the $V$-parameter of the 4-core fibers becomes higher, the coupling length of the supermodes obtained for a certain core separation increases, as demonstrated by Fig. 3(b) and (c). However, the behaviour of $L_{c}$ versus $q_{t o t}$ for different $d$ values is the same previously described. Notice that, as the $V$-parameter increases, the core-to-core distance providing negligible effects of the total heat load on $L_{c}$ becomes shorter, being around $35 \mu \mathrm{m}$ for the fibers with $V$-parameter $=2.4$. Moreover, under the same thermal effects, the optical coupling between SMs guided by 4-core fibers with higher $V$-parameter becomes weaker for shorter core separations. In fact, both the SM couples can be considered optically decoupled in the fiber with $V$-parameter $=2.4$ provided that $d \geq 50 \mu \mathrm{m}$, while in the 4-core fiber with intermediate $V$-parameter value, only Mode 1 and Mode 4 are still optically coupled, regardless of the total heat load $q_{t o t}$, when the core-to-core distance is $50 \mu \mathrm{m}$. Starting from these considerations, the impact of the generated heat load on the effective area $A_{\text {eff }}$ and the single-mode regime of 4-core fibers with optically coupled and decoupled cores are analyzed in the following Subsections.

\section{A. Effective area}

The fundamental SM $A_{\text {eff }}$ versus $d$ when the total heat load $q_{\text {tot }}$ is 20, 100 and $200 \mathrm{~W} / \mathrm{m}$ is shown in Fig. 4(a), (b) and (c) for the 4-core fibers with $V$-parameter equal to $1.735,2.2$ and 2.4 , respectively. Notice that, being the same the effective area behaviour of Mode 2 and 3, the single label Mode 2/3 is adopted also for this figure. The black horizontal dashed lines represent 4 times the value of the fundamental mode $A_{\text {eff }}$ in single-core fibers with the same $V$-parameter value 

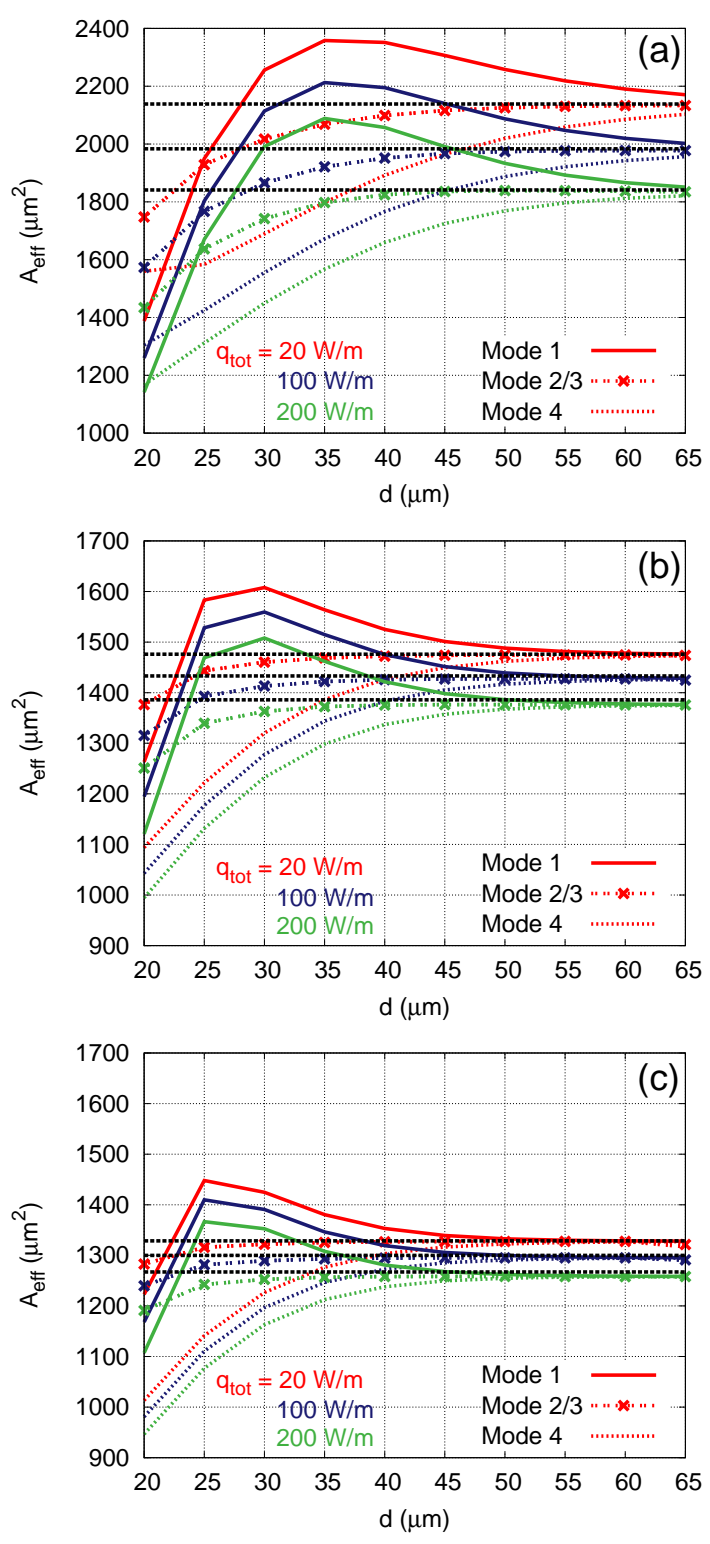

Fig. 4. Effective area of fundamental SMs as a function of distance $d$ for $q_{\text {tot }}=20,100$ and $200 \mathrm{~W} / \mathrm{m}$ generated in the 4-core fibers with $V$-parameter equal to (a) 1.735, (b) 2.2 and (c) 2.4. Horizontal black dashed lines represent four times the effective area of the corresponding single-core fiber.

when a quarter of the considered $q_{t o t}$, that is 5,25 and 50 $\mathrm{W} / \mathrm{m}$, is generated in the $\mathrm{Yb}$-doped core. By comparing the 4-core fiber SM $A_{\text {eff }}$ with these reference lines, it is possible to evaluate the potential advantages provided by the presence of the four cores in the fiber cross-section, due to the SM guidance, when the separation $d$ changes and different heating regimes are considered.

Regardless of the $V$-parameter value, Mode $4 A_{\text {eff }}$ always increases with the core-to-core separation $d$ in the range considered for the simulations. In particular, the lowest values are obtained when the distance among the cores is the shortest one, that is $20 \mu \mathrm{m}$. On the contrary, for largest $d$, Mode 4 $A_{\text {eff }}$ tends to reach the black line limit. It is interesting to notice that the Mode 2/3 effective area is the least affected by the core-to-core distance. Its behaviour versus $d$ is similar to the one of Mode $4 A_{\text {eff }}$, but with higher values when the four cores in the fiber cross-section are optically coupled, that is for a short distance among them. Moreover, for a fixed $V$-parameter value, the Mode 2/3 effective area approaches the single-core fiber reference line for a smaller core-to-core separation with respect to the Mode 4 one. Except for the fibers with core separation $d<25 \mu \mathrm{m}$, Mode 1 has the highest effective area value. Starting from the lowest values for $d=$ $20 \mu \mathrm{m}$, Mode $1 A_{\text {eff }}$ increases with the distance among the cores, until it reaches a maximum for the 4-core fiber with $d \simeq 35,30,25 \mu \mathrm{m}$ as $V$-parameter increases from 1.735 to 2.4. Then, for wider core-to-core separations, the effective area of the first SM decreases and tends to the reference line. In summary, when the distance among the four cores widens and they become optically decoupled, all the SMs tends to have the same effective area, very similar to the black line limit.

Notice that only the Mode $1 A_{\text {eff }}$ becomes higher than four times the single-core effective area for a certain $V$-parameter value. This happens in a $d$ range which decreases as the refractive index difference between core and cladding becomes higher. In fact, as the $V$-parameter changes from 1.735 to 2.4 , the core distance $d$, which corresponds to all the SM effective area values approaching the reference limit, becomes shorter. Moreover, also the maximum value of the effective area provided by the 4-core fibers is affected by the refractive index profile. In particular, the advantage provided by taking into account Mode $1 A_{\text {eff }}$ with respect to the reference line decreases from $220 \mu \mathrm{m}^{2}$ to $132 \mu \mathrm{m}^{2}$ and, finally, to $120 \mu \mathrm{m}^{2}$ for the 4-core fibers with $V$-parameter equal to 1.735, 2.2 and 2.4 , respectively, when the lowest $q_{t o t}$ value is considered. As expected, the $A_{\text {eff }}$ of all the SMs decreases at higher heat load values, regardless of $d$. It is important to underline that this effect is stronger for 4-core fibers with low $V$-parameter. In fact, as $q_{t o t}$ increases from $20 \mathrm{~W} / \mathrm{m}$ to $200 \mathrm{~W} / \mathrm{m}$, the maximum effective area decreases from $2358 \mu \mathrm{m}^{2}$ to 2088 $\mu \mathrm{m}^{2}$ for the fibers with $V$-parameter $=1.735$, and from 1448 $\mu \mathrm{m}^{2}$ to $1367 \mu \mathrm{m}^{2}$ for the ones with $V$-parameter $=2.4$. Finally, a comparison can be done with the $A_{\text {eff }}$ results for the corresponding dual-core fibers, shown in [16]. Notice that the $A_{e f f}$ behaviour versus the core-to-core separation $d$ of the even SM in dual-core fibers is analogous to the one of Mode 1 of 4-core fibers. The same comment applies for the odd SM and for Mode 4 of the fibers with two and four cores, respectively. Notice that, for a fixed $V$-parameter value, the maximum effective area, provided by the even SM and by Mode 1 in 2- and 4-core fibers, respectively, is obtained for the same core distance $d$, regardless of the total heat load. Moreover, the maximum $A_{\text {eff }}$ reached in 4-core fibers is almost twice the one obtained when there are only two $\mathrm{Yb}$ doped cores in the fiber cross-section, provided that the same $q_{t o t}$ is considered. A further increase of the core number in the fiber cross-section is expected to be advantageous in terms of supermode effective area.

\section{B. Single-mode regime}

The comparison of the single-mode regime of multicore fibers with two and four Yb-doped cores has been performed 
as their distance $d$ increases from $20 \mu \mathrm{m}$ to $65 \mu \mathrm{m}$, that is passing from an optically coupled condition to the decoupled one. Fig. 5 reports the core overlap integral $\Gamma$ of the supermodes of fibers with the intermediate $V$-parameter value among the ones considered in this analysis, that is 2.2 , when the total heat load is (a) 20, (b) 40, (c) 100 and (d) $200 \mathrm{~W} / \mathrm{m}$. Notice that, being $q_{t o t}$ the same for both the fiber types, in each core of the dual-core fibers there is twice the heat load of the 4-core fiber one. As it is explained in the legend of Fig. 5(a), red curves represent the values of $\Gamma$ of dual-core fiber SMs, while the blue ones refer to the results for 4-core fiber modes. It is important to underline that $\Gamma$ of even SM and of Mode 1 in 2and 4-core fibers, respectively, have a similar behaviour, as it has been already observed for the effective area. In particular, for the shortest core distance, $d=20 \mu \mathrm{m}, \Gamma$ has almost the same value for the even SM and for Mode 1, being 0.807 and 0.810 , respectively, when $q_{t o t}=20 \mathrm{~W} / \mathrm{m}$, as shown in Fig. 5(a). When the core-to-core separation becomes larger, the overlap integral of both even SM and Mode 1 reaches a minimum, around 0.772 and 0.753 , respectively, for $d=$ $25 \mu \mathrm{m}$. Then, $\Gamma$ increases, approaching an almost constant value for $d>50 \mu \mathrm{m}$, which is the same, around 0.792 , for the even supermode of the dual-core fiber and for Mode 1 of the 4-core fiber. Notice that the behaviour versus the core separation $d$ is the same even for higher value of the total heat load. However, as $q_{t o t}$ increases, the core overlap integral of the 2-core fiber even SM becomes higher than the one of the 4-core fiber Mode 1, especially for $d$ in the range between $25 \mu \mathrm{m}$ and $35 \mu \mathrm{m}$. In fact, as shown in Fig. 5(b)-(d), when the thermal effects are stronger, the minimum of the core overlap integral of the even SM increases to 0.774, 0.780 and 0.802 for $q_{t o t}=40,100$ and $200 \mathrm{~W} / \mathrm{m}$, respectively, while the one of Mode 1 is almost unaffected by the $q_{t o t}$ increase, being $0.753,0.756$ and 0.758 , respectively. Notice that also for optically decoupled Yb-doped cores, that is for $d>50 \mu \mathrm{m}$, the difference between the $\Gamma$ values of the even SM and of Mode 1 is no longer negligible, since it is around 0.02. Finally, being the core overlap integral of the even SM the highest of the two, regardless of the core-to-core separation $d$, when the total heat load increases, it is possible to conclude that, as the thermal effects get stronger, the supermode behaviour in the multicore fibers is more affected by the head load generated in each core, which is higher in the dual-core fibers, rather than by $q_{t o t}$.

Similarities in the core overlap integral behaviour can be found also by taking into account the odd supermode of the dual-core fiber and Mode 4 of the 4-core one. As demonstrated by Fig. $5, \Gamma$ values for these modes are higher than the ones for the supermodes previously analyzed, when there is a strong optical coupling among the doped cores, that is for $d=20 \mu \mathrm{m}$. In fact, the core overlap integral is 0.818 and 0.837 for the odd SM and Mode 4, respectively, when the total heat load is $20 \mathrm{~W} / \mathrm{m}$. Differently from the previous couple of supermodes, $\Gamma$ reaches a maximum value for the odd SM and for Mode 4 when the core-to-core distance is equal to $25 \mu \mathrm{m}$. After that, for larger core separations, it decreases, approaching an almost constant value. It is important to underline that, for optically decoupled cores, when $d$ is larger than $50 \mu \mathrm{m}$, even and odd SMs in dual-
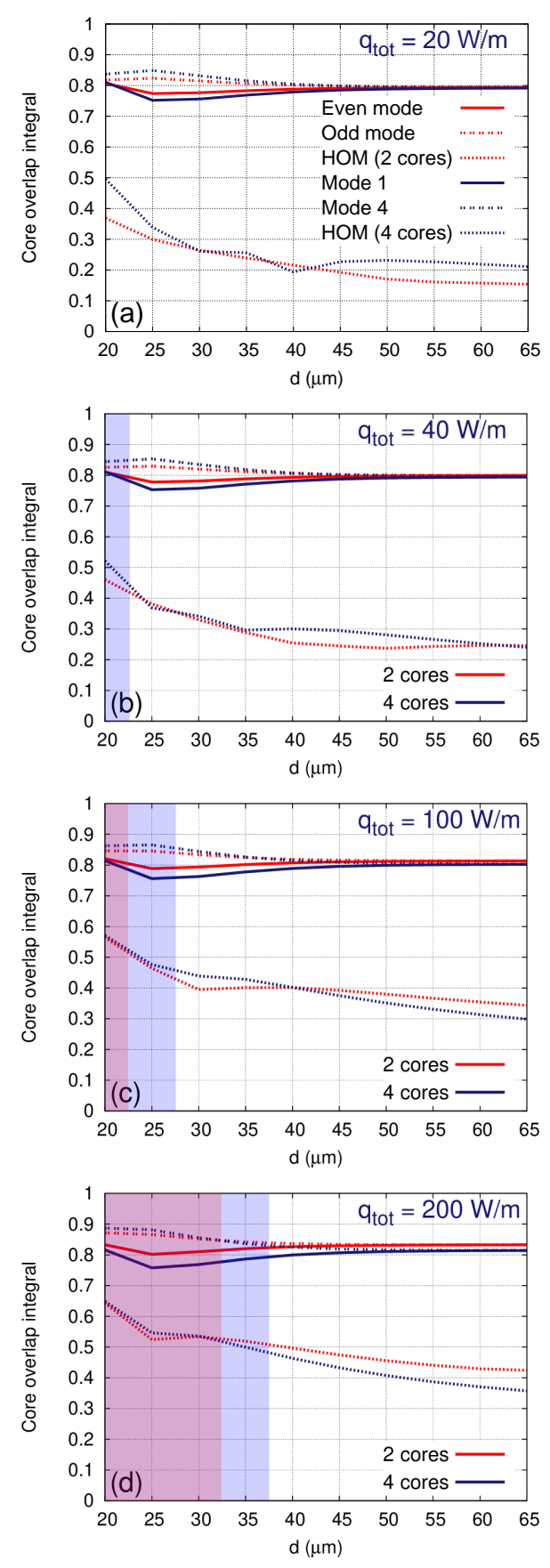

Fig. 5. Core overlap integral difference between fundamental SMs and most detrimental HOM as a function of distance $d$ for $q_{t o t}$ of (a) 20, (b) 40, (c) 100 and (d) $200 \mathrm{~W} / \mathrm{m}$ generated in 2- and 4-core fibers with $V$-parameter $=2.2$. Light-red and light-blue regions correspond to $d$ values providing multimode 2- and 4-core fibers, respectively.

core fibers have the same core overlap integral value [16], as well as Mode 1 and Mode 4 in 4-core fibers. Notice that, if $q_{t o t}$ increases from $20 \mathrm{~W} / \mathrm{m}$ to $200 \mathrm{~W} / \mathrm{m}$, the core overlap integral of both the odd SM and Mode 4 becomes higher, as expected. Moreover, the latter has a tighter confinement in the doped cores when they are optically coupled, especially for $d<35$ $\mu \mathrm{m}$. The opposite is true for large core separations, as it has been already underlined for the other couple of fundamental supermodes guided in 2- and 4-core fibers. 


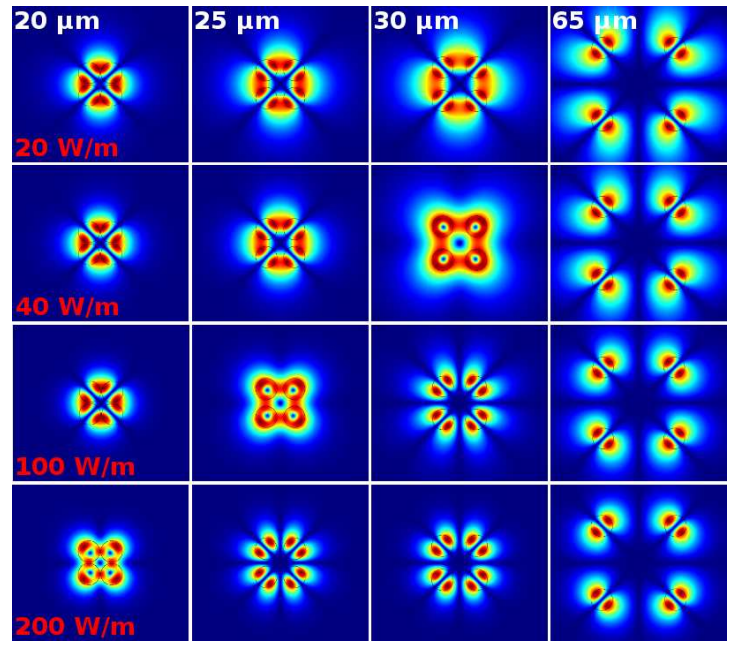

Fig. 6. Electric field modulus distribution of the most detrimental HOM in 4-core fibers with $V$-parameter $=2.2$ for a core separation $d=20,25,30$ and $65 \mu \mathrm{m}$ (column), when $q_{t o t}=20,40,100$ and $200 \mathrm{~W} / \mathrm{m}$ (row).

Finally, in order to evaluate the single-mode behaviour, the criterion based on the overlap integral difference $\Delta \Gamma$ between the fundamental SM and the most detrimental HOM, that is the one with the highest overlap integral on the four doped-cores, has been adopted [24]. In particular, $\Delta \Gamma$ should be higher than 0.3 to obtain the effective HOM suppression and, thus, the fiber operation in the single-mode regime. Fig. 5 reports with dotted lines the highest $\Gamma$ values calculated for the eight HOMs of the dual-core fibers [16], [22] and the sixteen ones of the 4-core fibers [22], as a function of the core-to-core distance $d$. The electric field modulus distribution of the most detrimental HOM for the 4-core fiber with $V$-parameter $=2.2$ is reported in Fig. 6, from left to right, for a core-to-core distance $d=20,25,30$ and $65 \mu \mathrm{m}$. The field evolution as the total heat load increases from 20 to $200 \mathrm{~W} / \mathrm{m}$ is shown, for a fixed core separation, in each column, from the top down. Notice that, when the cores are optically decoupled, the HOM with the highest overlap integral is the same, regardless of $q_{t o t}$, which causes only a tighter field confinement, as it increases. On the contrary, the most detrimental HOM changes with the total heat load when there is a significant optical coupling among the cores. Moreover, a different behaviour is obtained as a function of the core distance. However, it is important to underline that the most detrimental HOM tends to become the one obtained for optically decoupled cores as $d$ becomes larger. This happens for a shorter core separation as $q_{t o t}$ increases, that is for $d=45,40,30$ and $25 \mu \mathrm{m}$ for a total heat load of $20,40,100$ and $200 \mathrm{~W} / \mathrm{m}$, respectively.

Fig. 5(a) demonstrates that, for the lowest total heat load, the core overlap integral of the 4-core fiber HOM is significantly higher than the one of the dual-core fiber mode when the $\mathrm{Yb}$-doped cores are optically coupled, that is for $d<30$ $\mu \mathrm{m}$. For an intermediate coupling situation among the cores, that is for $d$ between $30 \mu \mathrm{m}$ and $45 \mu \mathrm{m}$, the $\Gamma$ values for the most detrimental HOM are almost the same for both the fiber types, regardless the core number in the cross-section. When the core-to-core separation is larger than $45 \mu \mathrm{m}$, the core overlap integral becomes almost constant for both the modes, but it is higher, around 0.22 , for the HOM guided in the 4-core fibers. When the thermal effects become stronger, as demonstrated by Fig. 5(b)-(d), the range of $d$ values where $\Gamma$ of the 4-core fiber HOM is higher than the one of the dualcore fiber mode shrinks more and more. In particular, for the highest total heat load, the most detrimental HOM of both the fiber types has the same confinement in the Yb-doped cores when they are optically coupled, that is for $d<35 \mu \mathrm{m}$. For larger core-to-core separation, the dual-core fiber HOM has the highest core overlap integral, which decreases with $d$, being 0.519 for $d=35 \mu \mathrm{m}$ and 0.424 for $d=65 \mu \mathrm{m}$. Notice that a similar decreasing behaviour is obtained for $\Gamma$ of the 4-core fiber $\mathrm{HOM}$, which changes from 0.5 to 0.358 when $d$ varies from $35 \mu \mathrm{m}$ to $65 \mu \mathrm{m}$.

By evaluating $\Delta \Gamma$, it is possible to identify a range of core-tocore distance values corresponding to a multi-mode behaviour for 2- and 4-core fibers at $1032 \mathrm{~nm}$ when the total heat load $q_{t o t}$ is generated in the $\mathrm{Yb}$-doped cores. This range of $d$ values is graphically represented in Fig. 5 with light-red and light-blue shadowed areas for fibers with two and four cores in the cross-section, respectively. Notice that the differential overlap integral $\Delta \Gamma$ is calculated taking into account, as the fundamental mode, the even SM for dual-core fibers and Mode 1 for 4-core ones, since this choice represents the worst-case for the single-mode regime. Simulation results in Fig. 5(a) show that a total heat load of $20 \mathrm{~W} / \mathrm{m}$ is not enough to modify the single-mode behaviour of both the multicore fiber types, regardless to the core separation. On the contrary, when thermal effects becomes higher, Fig. 5(b)-(d) demonstrate that they have a stronger influence on the single-mode regime of 4core fibers rather than on the one of dual-core fibers, when the same $q_{t o t}$ is considered, especially for short core separations. In particular, for 4-core fibers a total heat load of $40 \mathrm{~W} / \mathrm{m}$ is sufficient to loose the single-mode behaviour when the cores are optically coupled, that is for $d<25 \mu \mathrm{m}$. The $d$ range corresponding to a multimode regime enlarges as the thermal effects becomes more significant, being for $d<30 \mu \mathrm{m}$ and $d<40 \mu \mathrm{m}$ when $q_{t o t}$ is equal to $100 \mathrm{~W} / \mathrm{m}$ and $200 \mathrm{~W} / \mathrm{m}$, respectively. Notice that dual-core fibers demonstrate to be more robust to thermal effects than 4-core ones. In fact, their single-mode behaviour is affected by the heat load only when $q_{\text {tot }}$ is higher than $40 \mathrm{~W} / \mathrm{m}$. Moreover, under the same total heat load, the $d$ range corresponding to the multimode regime is always smaller than the one obtained for 4-core fibers, being $d<25 \mu \mathrm{m}$ and $d<35 \mu \mathrm{m}$ when $q_{t o t}$ is equal to $100 \mathrm{~W} / \mathrm{m}$ and $200 \mathrm{~W} / \mathrm{m}$, respectively.

In summary, it is important to underline that none of the two multicore fiber types considered in the present analysis becomes multimode for large core-to-core separations. It means that, when the cores are optically decoupled, thermal effects are less effective in modifying the confinement of the supermodes, especially of the higher-order ones, in the $\mathrm{Yb}$ doped cores. Promising results on the single-mode behaviour of Yb-doped multi-core fibers with 9 and 16 optically decoupled cores have been recently demonstrated, provided that the core diameter is properly selected [25]. On the contrary, the influence of the generated head load is strong when there is a 
significant optical coupling among the cores. In this condition, the core number has an important influence on the multicore fiber single-mode regime, which becomes higher and higher as the total heat load increases.

\section{CONCLUSION}

The influence of thermal effects on the guiding properties of multicore fibers with four Yb-doped cores in different optical coupling conditions has been numerically analyzed with the finite element method. Through the comparison with the properties of dual-core fibers with the same core dimension and refractive index profile, guidelines have been obtained for the choice of the best core number in the fiber cross-section, in order to optimize the performances of high-power lasers and amplifiers. Results have demonstrated that, for a fixed value of the total heat load, 4-core fibers are more convenient than dualcore ones to enlarge the effective area. To maximize $A_{\text {eff }}$, it is advantageous to consider the fundamental supermode with the highest effective index, that is Mode 1, and a quite small core separation, in the range between $25 \mu \mathrm{m}$ and $35 \mu \mathrm{m}$. In this condition, it is possible to exploit also the intrinsic coherence property due to the light propagation in supermodes, which can be automatically maintained along the multicore fiber amplifier without any feedback system. As expected, strong thermal effects cause an $A_{\text {eff }}$ decrease, due to the tighter supermode field confinement in the doped cores. However, the singlemode regime of 4-core fibers is more sensitive to thermal effects than the one of dual-core fibers, especially when the core-to-core distance is small. In fact, if the cores are optically coupled and the total heat load is high, it is better to select multicore fibers with few doped cores in the cross-section, in order to maintain the single-mode behaviour. On the contrary, it has been demonstrated that, when the cores are far enough to be optically decoupled, that is for $d \geq 50 \mu \mathrm{m}$, the singlemode regime can be preserved even for a higher core number in the presence of strong thermal effects. In summary, the choice of 4-core fibers with a core separation larger than 50 $\mu \mathrm{m}$, regardless of the $V$-parameter value, represents a good solution to obtain an improved behaviour both in terms of effective area, which is four times larger than the single-core one, and of single-mode behaviour, even under severe heat load conditions.

\section{REFERENCES}

[1] B. J. Puttnam, G. Rademacher, R. S. Lus, T. A. Eriksson, W. Klaus, Y. Awaji, N. Wada, K. Maeda, S. Takasaka, and R. Sugizaki, "High DataRate and Long Distance MCF Transmission With 19-Core C+L band Cladding-Pumped EDFA," Journal of Lightwave Technology, vol. 38, no. 1, pp. 123-130, 2020.

[2] J. D. Downie, X. Liang, and S. Makovejs, "Examining the Case for Multicore Fibers in Submarine Cable Systems Based on Fiber Count Limits," IEEE Journal of Selected Topics in Quantum Electronics, vol. 26, no. 4, pp. 1-9, 2020.

[3] H. H. Diamandi, Y. London, A. Bergman, G. Bashan, J. Madrigal, D. Barrera, S. Sales, and A. Zadok, "Opto-Mechanical Interactions in Multi-Core Optical Fibers and Their Applications," IEEE Journal of Selected Topics in Quantum Electronics, vol. 26, no. 4, pp. 1-13, 2020.

[4] A. V. Andrianov, N. A. Kalinin, E. A. Anashkina, O. N. Egorova, D. S. Lipatov, A. V. Kim, S. L. Semjonov, and A. G. Litvak, "Selective Excitation and Amplification of Peak-Power-Scalable Out-ofPhase Supermode in Yb-Doped Multicore Fiber," Journal of Lightwave Technology, vol. 38, no. 8, pp. 2464-2470, 2020.
[5] H.-J. Otto, A. Klenke, C. Jauregui, F. Stutzki, J. Limpert, and A. Tünnermann, "Scaling the mode instability threshold with multicore fibers," Optics Letters, vol. 39, no. 9, pp. 2680-2683, May 2014.

[6] J. Ji, S. Raghuraman, X. Huang, J. Zang, D. Ho, Y. Zhou, Y. Benudiz, U. B. Ami, A. A. Ishaaya, and S. Yoo, " $115 \mathrm{~W}$ fiber laser with an all solid-structure and a large-mode-area multicore fiber," Opt. Lett., vol. 43, no. 14, pp. 3369-3372, Jul 2018.

[7] C. Jauregui, J. Limpert, and A. Tünnermann, "High-power fibre lasers," Nature Photonics, vol. 7, no. 11, pp. 861-867, Nov 2013.

[8] T. Y. Fan, "Laser beam combining for high-power, high-radiance sources," IEEE Journal of Selected Topics in Quantum Electronics, vol. 11 , no. 3, pp. 567-577, 2005.

[9] A. Klenke, M. Müller, H. Stark, M. Kienel, C. Jauregui, A. Tünnermann, and J. Limpert, "Coherent Beam Combination of Ultrafast Fiber Lasers," IEEE Journal of Selected Topics in Quantum Electronics, vol. 24, no. 5, pp. 1-9, Sep. 2018.

[10] L. P. Ramirez, M. Hanna, G. Bouwmans, H. E. Hamzaoui, M. Bouazaoui, D. Labat, K. Delplace, J. Pouysegur, F. Guichard, P. Rigaud, V. Kermène, A. Desfarges-Berthelemot, A. Barthélémy, F. Prévost, L. Lombard, Y. Zaouter, F. Druon, and P. Georges, "Coherent beam combining with an ultrafast multicore Yb-doped fiber amplifier," Opt. Express, vol. 23, no. 5, pp. 5406-5416, Mar 2015.

[11] F. Prévost, L. Lombard, J. Primot, L. P. Ramirez, L. Bigot, G. Bouwmans, and M. Hanna, "Coherent beam combining of a narrow-linewidth long-pulse $\mathrm{Er}^{3+}{ }^{-}$-doped multicore fiber amplifier," Opt. Express, vol. 25, no. 9, pp. 9528-9534, May 2017.

[12] A. Klenke, M. Müller, H. Stark, F. Stutzki, C. Hupel, T. Schreiber, A. Tünnermann, and J. Limpert, "Coherently combined 16-channel multicore fiber laser system," Optics Letters, vol. 43, no. 7, pp. 15191522, Apr 2018.

[13] T. Eidam, C. Wirth, C. Jauregui, F. Stutzki, F. Jansen, H.-J. Otto, O. Schmidt, T. Schreiber, J. Limpert, and A. Tünnermann, "Experimental observations of the threshold-like onset of mode instabilities in high power fiber amplifiers," Opt. Express, vol. 19, no. 14, pp. 13 218-13 224, Jul 2011.

[14] J. Lægsgaard, F. Poli, A. Cucinotta, and S. Selleri, "Theory of thermooptic instabilities in dual-core fiber amplifiers," Opt. Lett., vol. 43, no. 19, pp. 4775-4778, Oct 2018.

[15] _ - "Static and dynamic mode instabilities in dual-core fiber amplifiers," J. Opt. Soc. Am. B, vol. 36, no. 3, pp. 757-767, Mar 2019.

[16] F. Poli, J. Lægsgaard, A. Cucinotta, and S. Selleri, "Thermal Effects on Modal Properties of Dual-Core Yb-Doped Fibers," J. Lightwave Technol., vol. 37, no. 4, pp. 1075-1083, Feb 2019.

[17] D. C. Brown and H. J. Hoffman, "Thermal, stress, and thermo-optic effects in high average power double-clad silica fiber lasers," IEEE Journal of Quantum Electronics, vol. 37, no. 2, pp. 207-217, Feb. 2001.

[18] A. Klenke, M. Müller, H. Stark, F. Stutzki, C. Hupel, T. Schreiber, A. Tünnermann, and J. Limpert, "Coherently combined femtosecond pulses from a multicore fiber amplifier," in Laser Congress 2018 (ASSL). Optical Society of America, 2018, p. AM2A.7.

[19] N. K. Fontaine, J. E. A. Lopez, H. Chen, R. Ryf, D. Neilson, A. Schulzgen, J. C. A. Zaharias, R.-J. Essiambre, H. Sakuma, T. Hasegawa, T. Nakanishi, T. Hayashi, and R. Amezcua-Correa, "Coupled-core optical amplifier," in Optical Fiber Communication Conference Postdeadline Papers. Optical Society of America, 2017, p. Th5D.3.

[20] "Comsol Multiphysics ${ }^{\circledR}$ v. 5.0," www.comsol.com, COMSOL AB, Stockholm, Sweden.

[21] C. Xia, N. Bai, I. Ozdur, X. Zhou, and G. Li, "Supermodes for optical transmission," Opt. Express, vol. 19, no. 17, pp. 16653-16664, Aug 2011.

[22] C. Xia, M. A. Eftekhar, R. A. Correa, J. E. Antonio-Lopez, A. Schülzgen, D. Christodoulides, and G. Li, "Supermodes in Coupled Multi-Core Waveguide Structures," IEEE Journal of Selected Topics in Quantum Electronics, vol. 22, no. 2, pp. 196-207, Mar 2016.

[23] L. Rosa, S. H. Pallangal, F. Poli, S. Selleri, and A. Cucinotta, "Mode phase variation and sensitivity to thermal load in three-core optical fibers," Journal of Lightwave Technology, vol. 38, no. 8, pp. 2400-2405, 2020.

[24] M. M. Jørgensen, S. R. Petersen, M. Laurila, J. Lægsgaard, and T. T. Alkeskjold, "Optimizing single mode robustness of the distributed modal filtering rod fiber amplifier," Opt. Express, vol. 20, no. 7, pp. 7263-7273, Mar 2012.

[25] L. Rosa, S. Mckee, L. Vincetti, F. Poli, S. Selleri, and A. Cucinotta, "Thermo-optic effects in multicore fibers for high-power lasers," in Fiber Lasers and Glass Photonics: Materials through Applications II, vol. 11357. SPIE, 2020, pp. $165-170$. 